\title{
High expression of suppressor of cytokine signaling 1 (SOCS1) associates with favourable responsiveness to neoadjuvant chemoradiotherapy in esophageal squamous cell carcinoma
}

Kai Xiong

Army Medical University

Yunfei Ye

Army Medical University

Jian Li

Army Medical University

Lin Lei

Army Medical University

He Xiao

Army Medical University

Xueqin Yang ( $\triangle$ yangxueqin@hotmail.com )

Army Medical University

\section{Research Article}

Keywords: ESCC, neoadjuvant therapy, responsiveness, SOCS1, ORAI3

Posted Date: May 7th, 2021

DOI: https://doi.org/10.21203/rs.3.rs-464237/v1

License: (c) (1) This work is licensed under a Creative Commons Attribution 4.0 International License.

Read Full License 


\section{Abstract}

Background: The responsiveness to preoperative neoadjuvant therapy in esophageal squamous cell carcinoma (ESCC) is significantly related to the surgical effect and long-term prognosis of patients. Biomarkers for predicting the effect of preoperative neoadjuvant therapy of ESCC are urgently needed in clinical practice. M2 macrophage in the tumor microenvironment (TME) has been confirmed to have a definite correlation with neoadjuvant therapy. However, the research on the potential functional genes of M2 macrophage has not been carried out. The purpose of this study is to systematically screen the potential functional genes of M2 macrophages and verify the correlation between the expression of screened genes and responsiveness to neoadjuvant therapy in ESCC.

Methods: The Cancer Genome Atlas (TCGA) database was used to screen the potential functional genes of M2 macrophages systematically. The correlation between the screened genes and responsiveness to neoadjuvant therapy in ESCC was analyzed in the training set GSE45670 and the validation set GSE104958. The correlation of the screened genes was confirmed in a cohort of 27 patients using immunohistochemistry (IHC).

Results: A total of $11 \mathrm{M} 2$ macrophage potential functional genes were screened out. The suppressor of cytokine signaling 1 (SOCS1) and ORAI calcium release-activated calcium modulator 3 (ORAI3) were selected for subsequent verification. The expression of SOCS1 and ORAI3 in 27 cases of ESCC was evaluated by the $\mathrm{H}$-score system. The result showed that the high expression of SOCS1 was significantly correlated with favourable responsiveness to preoperative neoadjuvant therapy (AUC $=0.830,95 \% \mathrm{Cl}$ : $0.647-1, P=0.006)$. No significant correlation was found between the expression of ORAI3 and neoadjuvant therapy in ESCC (AUC $=0.688,95 \% \mathrm{Cl}: 0.478-0.899, \mathrm{P}=0.117)$.

Conclusion: The high expression of SOCS1 in the local tumor microenvironment is significantly correlated with favourable responsiveness to neoadjuvant therapy in patients with ESCC, which can be used as a biomarker to predict the responsiveness to neoadjuvant therapy, and has certain clinical value.

\section{Introduction}

Esophageal cancer is the sixth most common tumor in the world [1]. The symptoms of patients with early esophageal cancer are not typical, and the disease is often in the middle and late stages when swallowing obstruction occurs. For patients with locally advanced esophageal cancer, preoperative neoadjuvant therapy is often used to reduce the tumor stage, and the following radical resection can significantly improve the R0 resection rate and improve the prognosis [2]. However, the responsiveness of neoadjuvant therapy varies from patient to patient, according to the disease stage, age, immune status and tumor heterogeneity [3]. Patients with poor responsiveness to neoadjuvant therapy suffer from not only difficulties in receiving effective treatment, but also unnecessary economic pressures. Therefore, it is crucial to predict the responsiveness to neoadjuvant therapy. Many studies have shown that tumor microenvironment (TME) is closely related to tumor treatment efficacy and prognosis $[4,5]$. TME, 
composed of immune cells and their subtypes, stromal cells, various small molecules and other components, is closely related to the occurrence, development and metastasis of tumor, and is an important part of tumor heterogeneity [6]. M2 macrophage is an important immune cell in TME, which can promote tumor chemoradiotherapy resistance. Previous studies have found that the higher the infiltration abundance of M2 macrophages in tumor tissue, the worse the responsiveness to preoperative neoadjuvant therapy, and the more difficult it is to achieve pathological remission [7]. Moreover, this study also found that the genes related to $M 2$ macrophage function are also related to the responsiveness to preoperative neoadjuvant therapy for ESCC [7]. Therefore, M2 macrophage function-related genes were

systematically screened using TCGA and Gene Expression Omnibus (GEO) database, and their correlation with the responsiveness to the preoperative neoadjuvant therapy of ESCC were analyzed in the training set and validation set. Verification was carried out using the preoperative specimens of ESCC in our hospital.

\section{Materials And Methods}

\section{Datasets and preprocessing}

TCGA Esophageal Cancer (ESCA) RNA expression matrix in the format of log2 $(x+1)$ transformed RSEM normalized count and phenotype file, as well as survival data, were downloaded from https://xenabrowser.net/datapages/. The series matrix files of two GEO datasets, GSE45670 and GSE104958, which contain RNA expression profiles and pathological responsiveness information, were obtained from the NCBI website (https://www.ncbi.nlm.nih.gov/geo/). The RNA expression profiles of GSE45670 and GSE104958 were log2 scale. The only probe set was selected to represent the expression level of corresponding genes based on the maximum interquartile range (IQR). All probe sets were annotated into ENTREZID. There were 11854 genes retained common to all three cohorts.

The GSE45670 dataset included the biopsies from 28 patients with ESCCs who received neoadjuvant chemoradiotherapy (nCRT) and surgery, in which 11 cases reached pathological complete remission (pCR) and the other 17 cases did not. All 41 tissue samples in the GSE104958 dataset were biopsies during the endoscopic examination before administering the first course of neoadjuvant chemotherapy with docetaxel, cisplatin and 5-fluorouracil, in which 10 cases reached pCR.

\section{Evaluation of abundance of immune cells infiltrating into tumor tissue}

MCP-counter was used to evaluate the abundance of 13 cell types including NK cells, B cells, CD8 T cells, endothelial cells, fibroblasts, etc. The abundance of 13 cell types was represented as log2 geometric mean of these immune cell transcriptomic markers called MCPcounter score. Signatures of macrophage M1 (CXCL11, ID01, CCL19, CXCL9, PLA1A, LAMP3, CCR7, APOL6, CXCL10) and M2 (DPEP2, CLEC4A, EGR2, CLEC10A, MS4A6A, CCL13, TREM2, CD180, RNASE6, NCF2, CD209) were extracted from the reference matrix of CIBERSORT (originally published material NIHMS670442-supplement-2) according to 
maximum expression level [8]. The abundance of M1 and M2 was represented as the average expression value of signature genes.

\section{Development of pathological response predicting model}

To identify genes that influence the function of M2 on responsiveness to nCRT, an approach that comprised the two main steps was adopted. Firstly, candidate genes that potentially influence the function of M2 were identified with the method similar to Peng's approach [9]. A total of 79 patients had squamous cell carcinoma with $\mathrm{R} 0$ resection and had a complete record of disease-specific survival (DSS) intervals and censored events in the TCGA ESCA data set. The baseline information for this cohort of TCGA is summarized in Table 1. Cox regression analysis had shown that the abundance of M2 macrophage was strongly associated with DSS in these 79 patients $(\mathrm{HR}=4.562,95 \% \mathrm{Cl}: 1.539-13.52, P$ $=0.00618$, Supplementary Table 1). A linear model hazard $=a \times M 2+b \times V+d \times M 2 \times V+c$ was solved using Cox regression for DSS. In the above formula, $a, b$ and $d$ represent the regression coefficients of the abundance of M2 macrophage, gene expression level, and the interaction of M2 macrophage and gene expression level respectively. The regression analysis of 11854 genes showed that the three coefficients $(a, b$ and $c$ ) of 279 genes were significant (crude $P<0.05$, Supplementary Table 2). Hence, 279 genes were selected as potential functional genes of M2 macrophages. 
Table 1

Clinical characteristics of ESCC patients from TCGA database

\begin{tabular}{|ll|}
\hline Variable & N(\%) or median(IQR) \\
\hline Age, median(IQR) & $57(50.5-62)$ \\
\hline Gender & \\
\hline Male & $67(84.81 \%)$ \\
\hline Female & $12(15.19 \%)$ \\
\hline Smoking history & \\
\hline Yes & $43(54.43 \%)$ \\
\hline NO & $36(45.57 \%)$ \\
\hline Alcohol history & \\
\hline Yes & $57(72.16 \%)$ \\
\hline NO & $20(25.31 \%)$ \\
\hline Unclear & $2(2.53 \%)$ \\
\hline Tumor central location & \\
\hline Middle & $36(45.57 \%)$ \\
\hline Distant & $39(49.37 \%)$ \\
\hline Proximal & $3(3.80 \%)$ \\
\hline Unclear & $1(1.26 \%)$ \\
\hline Pathologic stage & $3(37.97 \%)$ \\
\hline Stage I & $3(1.26 \%)$ \\
\hline Stage II & \\
\hline Stage III & $3(3.79 \%)$ \\
\hline Stage IV & \\
\hline pN stage & \\
\hline pN (-) & \\
\hline pN (+) & \\
\hline pNX & \\
\hline pT stage & \\
\hline
\end{tabular}




\begin{tabular}{|ll|}
\hline Variable & $\mathbf{N}(\%)$ or median(IQR) \\
\hline pT1 & $8(10.13 \%)$ \\
\hline pT2 & $31(39.24 \%)$ \\
\hline pT3 & $38(48.10 \%)$ \\
\hline pT4 & $2(2.53 \%)$ \\
\hline OS time months, median(IQR) & $13(11.55-18.73)$ \\
\hline DSS time months, median(IQR) & $13(11.55-18.73)$ \\
\hline
\end{tabular}

In the GSE45670 dataset, the ratio of 279 potential functional genes to the abundance of M2 macrophage genes was used as the candidate variable, and the Least Absolute Shrinkage and Selection Operator (LASSO) regression was used to construct the classifier predictive of pCR. Among the 279 potential functional genes, 11 genes were finally selected with the minimum $\lambda$ value. A weighted linear model was used as the predictive score for pathological response. However, to further verify the genes in the clinical setting, only the first two genes with the largest absolute regression coefficient were selected for subsequent verification, including suppressor of cytokine signaling 1 (SOCS1) and ORAI calcium release-activated calcium modulator 3 (ORAI 3). All the above analysis was done with R language (Version 3.6.2). The flow chart of the analysis was shown in Fig. 1.

\section{Specimen collection}

A total of 27 paraffin-embedded specimens of ESCC were collected before neoadjuvant therapy in our hospital from January 2014 to December 2019. Inclusion criteria were: (1) patients had a preoperative pathological diagnosis of ESCC; (2) nCRT were planed instead of surgical treatment after clinician evaluation; (3) patients had no previous chemoradiotherapy; (4)patients had no hematological diseases; (5) patients with complete clinical and follow-up data. Exclusion criteria were: (1) patients who could not complete nCRT or quit halfway; (2) patients with multiple organ dysfunction such as blood system, liver, and spleen; (3) patients who had other types of tumor or had distant metastasis. There are three main chemotherapy regimens: (1) DP regimen: Docetaxel $60-75 \mathrm{mg} / \mathrm{m} 2$ was given intravenously on the first day, and nedaplatin $75-80 \mathrm{mg} / \mathrm{m} 2$ on the second day. (2) TP Regimen: Paclitaxel $135-175 \mathrm{mg} / \mathrm{m} 2$ on the first day, nedaplatin $75-85 \mathrm{mg} / \mathrm{m} 2$ or lobaplatin $30-35 \mathrm{mg} / \mathrm{m} 2$ on the second day. (3) Other regimens: Docetaxel $60-75 \mathrm{mg} / \mathrm{m} 2$ on the first day, nedaplatin $75-80 \mathrm{mg} / \mathrm{m} 2$ on the second day, and tegafur $600-700 \mathrm{mg} / \mathrm{m} 2$ on day $1-5$, once a day. The treatment contained 2 cycles and 21 days every cycle. The total radiation dose was 41.4-50 Gy which was divided into 20-24 times. The patient information of the collected specimens was sorted out, mainly including gender, age, tumor location, staging, remission, etc.

\section{Evaluation of responsiveness to nCRT}

lodine water angiography and chest enhanced CT were used to record the changes of lesion size before and after neoadjuvant therapy. RECIST solid tumor efficacy evaluation standard 1.1 was used to evaluate 
the responsiveness to neoadjuvant chemoradiotherapy[10]: complete remission (CR): the target lesion disappeared; partial remission (PR): the sum of the longest single diameter of the lesion decreased by more than $30 \%$; progression (PD): the sum of the longest single diameter of the lesion increased by more than $20 \%$ or new lesions appeared; stability (SD): the sum of the longest single diameter of the lesion decreased but did not reach PR or increased but did not reach PD.

\section{Immunohistochemistry}

Paraffin-embedded specimens of ESCC were sectioned with a thickness of $3 \mu \mathrm{m}$. The slices were baked at $60{ }^{\circ} \mathrm{C}$ for $2 \mathrm{~h}$ and dewaxed in xylene for 15 minutes. $3 \%$ hydrogen peroxide was used to remove endogenous peroxidase activity. SOCS1 antibody (ab62584, Abcam antibody, USA, 1:250), ORAl3 antibody (ab254260, Abcam antibody, USA, 1:1000), and CD163 antibody (ZM-0428, Beijing Zhongshan Goldenbridge Biotechnology Co. Ltd, working solution) were incubated overnight at $4{ }^{\circ} \mathrm{C}$. Then, the combined second antibody (HRP labeled anti-mouse IgG + AP labeled anti-rabbit IgG, ds-0003, Beijing Zhongshan Goldenbridge Biotechnology Co. Ltd) was incubated at $37{ }^{\circ} \mathrm{C}$ for $30 \mathrm{~min}$. The DAB reagent kit (zli-9018, Beijing Zhongshan Goldenbridge Biotechnology Co. Ltd) was used for color development and hematoxylin staining. $\mathrm{H}$-score was used to evaluate the color intensity and the number of positive cells. The $\mathrm{H}$-score of each slice was obtained as follows: $\mathrm{H}$-score $=$ grade of positive degree $\times$ proportion of positive cells. The grade of positive degree was - (negative, grade 0$),+($ weak positive, grade 1$),++$ (medium positive, grade 2 ),+++ (strong positive, grade 3 ). The $\mathrm{H}$-score range was $0-300$. The $\mathrm{H}$-score valuation process was independently performed by two senior and experienced pathologists, who did not know the severity of the disease and the remission after treatment. If there was a large difference between the two scores, the third pathologist need to review the slices and the final $\mathrm{H}$-score was the average score of the two or three pathologists. The final efficacy prediction scores of the two antibodies were Score1 $=($ SOCS1 H-score $) /($ CD163 H-score $)$ and Score2 = (ORAl3 H-score) $/($ CD163 H-score), respectively.

\section{Statistical analysis}

SPSS 19.0 statistical software was used for statistical analysis. Receiver operating characteristic (ROC) curve analysis was used to evaluate the predictive efficacy of Score1 and Score2 in response to nCRT. In univariate analysis, the chi-square test was used to analyze the correlation between remission and gender, stage, chemotherapy regimen and other classification variables, and the Kruskal Wallis test was used to analyze the correlation between remission and age, Score1, Score2. $\mathrm{P}<0.05$ was considered statistically significant.

\section{Results}

\section{Screening and identification of potential function genes of M2 macrophages}


The linear risk model was constructed in TCGA data, and Cox regression was used for DSS. After regression analysis of 11854 genes, 279 genes had significant effects on M2 macrophage infiltration abundance, gene expression level, and the three regression coefficients of their interaction $(P<0.05)$. These $\mathbf{2 7 9}$ genes can be used as potential functional genes of M2 macrophages (Supplementary Table 2).

\section{Establishment and validation of the prediction model for nCRT}

The result of LASSO regression constructing the classifier predictive of pCR was presented in Supplementary Table 3. In the GSE45670 dataset, 11 genes were screened out from 279 potential functional genes to predict the response of neoadjuvant therapy of ESCC (Table 2). To meet the needs of actual detection, only SOCS1 and ORAI3 genes with larger regression coefficients were selected as followup validation genes. The sum of their regression coefficients multiplying by the ratio of the two genes expression to the abundance of M2 macrophage was calculated as the final index, namely Score for predicting pCR. Using the GSE45670 dataset as the training set, the area under the ROC curve (AUC) predicted by Score was 0.904 (95\% Cl: 0.792-1, P<0.001, Fig. 2a). The GSE104958 dataset was used as the validation set, and the Score was calculated by the same method. The AUC was $0.706(95 \% \mathrm{Cl}$ : 0.5033-0.9096) (Fig. 2b). The Score of pCR group was higher than that of the non-pCR group $(\mathrm{H}=3.775$, $P=0.052$, Fig. 3a). There was no significant difference in the infiltration abundance of M2 macrophages $(H=2.304, P=0.129)$ (Fig. $3 b)$. In this small sample size cohort, using the multiple gene expression ratios is more effective than using M2 abundance alone. To explore the correlation between the infiltration abundance of M2 macrophage and the expression of SOCS1 and ORAI3, MCPcounter score that represented the abundance of 10 immune cell types got from TCGA ESCA data set was used for regression analysis (Fig. 4a). The negative correlation between the expression ratio of SOCS1 to M2 macrophage, namely Score 1 and the infiltration abundance of M2 macrophage (Fig. $4 b$ ). A similar result of ORAI3 was represented in Fig. 4c. 
Table 2

List of genes finally selected by LASSO in the training set GSE45670

\begin{tabular}{|lll|}
\hline ENTREZID & Gene symbol & Coefficient \\
\hline 8651 & SOCS1 & 5.433 \\
93129 & ORAI3 & -3.089 \\
\hline 57720 & GPR107 & 3.029 \\
\hline 7102 & TSPAN7 & -2.807 \\
\hline 9953 & HS3ST3B1 & -2.082 \\
\hline 51517 & NCKIPSD & 1.799 \\
\hline 285195 & SLC9A9 & -1.440 \\
\hline 2760 & GM2A & 1.118 \\
\hline 2639 & GCDH & 1.051 \\
\hline 89801 & PPP1R3F & 0.665 \\
\hline 7511 & XPNPEP1 & 0.256 \\
\hline
\end{tabular}

\section{Clinical data and specimen collection}

A total of 27 patients with ESCC who received preoperative neoadjuvant therapy were collected, including 24 males and 3 females. All patients aged from 51 to 79 years old and median age was 64 years old. The lesions of 11 cases were located in the upper segment, 15 cases in the middle segment, 1 case in the lower segment. 11 cases were in stage II, 13 cases in stage III, 2 cases in stage IV. 15 cases underwent DP, 4 cases TP, and 8 cases other regimens. The responsiveness to nCRT was represented as follows: 0 cases of CR, 9 cases of PR, 15 cases of SD and 3 cases of PD (Table 3). The pathological specimens of 27 patients with ESCC were paraffin-embedded specimens of gastrointestinal endoscopic biopsy before nCRT.

\section{SOCS1 overexpression is associated with favourable responsiveness to preoperative neoadjuvant therapy}

To confirm the correlation between the expression of SOCS1 and ORAI3 in tumor tissues and preoperative neoadjuvant responsiveness, 27 cases of ESCC paraffin sections were stained by immunohistochemistry (Fig. 5). ROC curve was used to analyze the predictive effect of CD163 H-score, Score1 and Score2 on the efficacy of neoadjuvant therapy. The results showed that the AUC of CD163 H-score for predicting curative effect was $0.556(95 \% \mathrm{Cl}: 0.307-0.804, \mathrm{P}=0.643)$. Score 1 that reflected the expression of SOCS1, was significantly correlated with favorable responsiveness to preoperative neoadjuvant therapy (AUC $=0.830,95 \% \mathrm{Cl}: 0.647-1, \mathrm{P}=0.006$, Fig. $6 \mathrm{a}$ ). There was no significant correlation between Score2 that reflected the expression of ORAI3 and neoadjuvant therapy in ESCC (AUC $=0.688,95 \%$ Cl: $0.478-$ 
$0.899, P=0.117)$. Univariate analysis showed that $S c o r e 1$ in patients with $P R$ was significantly higher than that in patients with PD + SD $(P=0.006)(F i g .6 b)$. There was no significant difference in CD163 Hscore between patients with $P R$ and $P D+S D(H=0.216, P=0.642)$. Remission was significantly correlated with gender, stage $(P<0.05)($ Table 3$)$. 
Table 3

Univariate analysis of influencing factors of neoadjuvant therapy

\begin{tabular}{|c|c|c|c|c|}
\hline \multirow[t]{2}{*}{ Factors } & \multicolumn{2}{|c|}{ Response of neoadjuvant therapy } & \multicolumn{2}{|c|}{ Univariate analysis } \\
\hline & PR & $\mathrm{PD}+\mathrm{SD}$ & $\chi^{2} / H$ & $\mathbf{P}$ \\
\hline Number of patients & 9 & 18 & & \\
\hline \multicolumn{5}{|l|}{ Gender } \\
\hline Male & $6(66.67 \%)$ & $18(100 \%)$ & 6.750 & 0.009 \\
\hline Female & $3(33.33 \%)$ & $0(0)$ & & \\
\hline Age, median (IQR) & $68(67-70)$ & $61(55.75-69)$ & 3.531 & 0.06 \\
\hline \multicolumn{5}{|l|}{ cT stage } \\
\hline $\mathrm{CT}_{4}$ & $5(55.56 \%)$ & $0(0)$ & 12.273 & $<0.001$ \\
\hline $\mathrm{CT}_{2-3}$ & $4(44.44 \%)$ & $18(100 \%)$ & & \\
\hline \multicolumn{5}{|l|}{ cN stage } \\
\hline $\mathrm{CN}_{1-2}$ & $6(66.67 \%)$ & $8(44.44 \%)$ & 1.187 & 0.276 \\
\hline $\mathrm{CN}_{0}$ & $3(33.33 \%)$ & $10(55.56 \%)$ & & \\
\hline \multicolumn{5}{|l|}{ Clinical Stage } \\
\hline Stage III + IV & $8(88.89 \%)$ & $8(44.44 \%)$ & 4.909 & 0.027 \\
\hline Stage II & $1(11.11 \%)$ & $10(55.56 \%)$ & & \\
\hline \multicolumn{5}{|l|}{ Chemotherapy regimens } \\
\hline DP regimen & $6(66.67 \%)$ & $9(50.00 \%)$ & 0.171 & 0.918 \\
\hline TP regimen & $1(11.11 \%)$ & $4(22.22)$ & & \\
\hline Other regimens & $2(22.22 \%)$ & $5(27.78 \%)$ & & \\
\hline \multicolumn{5}{|l|}{ Tumor location } \\
\hline Upper & $4(44.44 \%)$ & $7(38.89 \%)$ & 2.285 & 0.130 \\
\hline Median & $4(44.44 \%)$ & $11(61.11 \%)$ & & \\
\hline Lower & $1(11.11 \%)$ & $0(0)$ & & \\
\hline CD163 H-score, median (IQR) & $220(200-240)$ & $210(170-227.5)$ & 0.216 & 0.642 \\
\hline Score1 & $0.22(0.14-0.28)$ & $0.16(0.12-0.24)$ & 7.591 & 0.006 \\
\hline Score2 & $0.21(0.20-0.25)$ & $0.10(0.06-0.18)$ & 2.462 & 0.117 \\
\hline
\end{tabular}




\section{Discussion}

Previous studies have confirmed a significant correlation between the infiltration degree of M2 macrophages in TME and the responsiveness to neoadjuvant therapy $[7,11]$. However, the mechanism of M2 macrophages participating in neoadjuvant therapy is still unclear. In this study, all the functional genes of M2 macrophages were systematically screened by the TCGA database for the first time. The functional genes, namely SOCS1 and ORAI3, which may participate in the preoperative neoadjuvant therapy of ESCC, were screened and verified by the GEO database.

In this study, there are two main reasons that Score was used to be the final evaluation criterion for predicting PCR in the GEO database. Firstly, Score was the sum of the two genes' regression coefficients multiplying by ratios of the expression of the two genes to the abundance of M2 macrophage genes, reflecting the relationship between the two genes and M2 macrophage better. Secondly, the expression of genes as the numerator in Score may be the leading factor of predicting the response of nCRT, not denominator. So it further indicated that the expression of potential genes had better predictive value than the abundance of M2 macrophage.

27 paraffin-embedded specimens of ESCC were used to verify the results of GEO database. We found no significant correlation between CD163 H-score and remission, which was consistent with the results of database analysis. Therefore, the predictive value of Score 1 for neoadjuvant therapy can reflect the correlation between SOCS1 expression in the local tumor microenvironment and the curative effect. Finally, we found that the high expression of SOCS1 is significantly correlated with the favorable responsiveness to preoperative neoadjuvant therapy for ESCC.

SOCS1, as a cell signal suppressor, is mainly involved in the regulation of two signaling pathways, namely Janus tyrosine kinase / signal transducer and activator of transcription (JAK/STAT) signaling pathway and toll-like receptors (TLR) signaling pathway $[12,13,14,15,16]$. In JAK / STAT signaling pathway, SOCS1 can interact with JAK kinase domains, such as Jak1, JAK2, Tyk2, to inhibit the activity of the kinase and affect the downstream STAT protein phosphorylation, leading to the inactivation of the signaling pathway and playing a negative feedback regulation role $[17,18,19]$. In the TLR signaling pathway, SOCS1 can interact with TLR adaptor proteins, such as MAL and IRAK, leading to their degradation, thereby inhibiting this signaling pathway $[16,17,20,21]$. As a tumor suppressor, SOCS1 gene promoter methylation leads to the inactivation of SOCS1 gene expression, which occurs in $53-71 \%$ of patients with pancreatic cancer and occurs in patients with acute myeloid leukemia and liver cancer $[22,23,24]$. McCormick found that in the signal pathway of IL-4 activating tyrosine phosphorylation of insulin receptor substrate-2 (IRS-2) to induce M2 macrophage polarization, SOCS1 protein can inhibit M2 macrophage differentiation by mediating IRS-2 ubiquitination and promoting protease degradation of phosphorylated IRS-2[25]. Whyte and Liu also found that SOCS1 was closely related to the differentiation of M2 macrophages [26, 27]. This study shows that SOCS1 can be used as a biomarker for the favorable responsiveness to preoperative neoadjuvant therapy for ESCC. The reason may be that SOCS1 can negatively regulate the differentiation of $\mathrm{M} 2$ macrophages in the tumor microenvironment, reduce the 
infiltration of M2 macrophages in the microenvironment, and thus reduce the immunosuppressive effect of M2 macrophages [28, 29, 30].

There are several limitations in this study: (1) The number of samples in training set GSE45670 and validation set GSE104958 is small, and the accuracy of the screened genes needs to be further studied and verified; (2) The number of paraffin-embedded specimens used for validation is small, and the results need to be further confirmed by expanding the sample size; (3) The ESCC specimens were from preoperative endoscopic biopsy, and the samples were taken from the surface of the tumor. So they may not reflect the whole tumor microenvironment. However, compared with the prediction efficiency of tumor microenvironment of preoperative endoscopic biopsy specimens and postoperative resection specimens in previous studies, it is found that the tumor microenvironment of preoperative endoscopic biopsy specimens can also better predict the prognosis and curative effect of patients [31, 32]. (4) The function of SOCS1 in M2 macrophage had not been studied, and further research is needed to verify the function.

In conclusion, the high expression of SOCS1 in the local tumor microenvironment is significantly related to the favorable responsiveness to neoadjuvant therapy in patients with ESCC, which can be used as a biomarker to predict the responsiveness to neoadjuvant therapy and has certain clinical value.

\section{Declarations}

\section{Ethics approval and consent to participate:}

The ethical approval for obtained from Ethics Committee of the Army Medical Center of the PLA. The committee's reference number was 2020\#91. Informed consent was obtained from all participants. All of those studies from databases previously were approved by their respective institutional review boards.

The need for informed consent of patients involved in three independent cohorts (TCGA ESCA, GSE45670, GSE104958) and our hospital was waived by the Ethics Committee of the Army Medical Center of the PLA. This study was conducted in accordance with the Declaration of Helsinki.

\section{Consent for publication:}

Not applicable

\section{Availability of data and materials:}

The datasets used and/or analysed during the current study are available from the corresponding author on reasonable request. Datasets of TCGA ESCA were downloaded from https://xenabrowser.net/datapages/. GSE45670 and GSE104958 were obtained from the NCBI website (https://www.ncbi.nlm.nih.gov/geo/).

\section{Competing interests:}

The authors declare that they have no competing interests. 


\section{Funding:}

Not applicable

\section{Author contributions}

$X Y, H X, K X$ and $Y Y$ performed design of the study. JL and LL retrieved all the data for ESCC patients information. $\mathrm{HX}$ and $\mathrm{KX}$ analyzed and interpreted the patient data. $\mathrm{KX}$ and $\mathrm{YY}$ completed the experiment and were major contributors in writing the manuscript. All authors read and approved the final manuscript.

\section{Acknowledgements:}

Not applicable

\section{References}

1. Sung H, Ferlay J, Siegel RL, et al. Global cancer statistics 2020: GLOBOCAN estimates of incidence and mortality worldwide for 36 cancers in 185 countries. CA Cancer J Clin. 2021 Feb 4. doi: 10.3322/caac. 21660 .

2. Shapiro J, van Lanschot JJB, Hulshof MCCM, et al. Neoadjuvant chemoradiotherapy plus surgery versus surgery alone for oesophageal or junctional cancer (CROSS): long-term results of a randomised controlled trial. Lancet Oncol. 2015 Sep;16(9):1090-1098. doi: 10.1016/S14702045(15)00040-6.

3. Anvari K, Aledavood SA, Toussi MS, et al. A clinical trial of neoadjuvant concurrent chemoradiotherapy followed by resection for esophageal carcinoma. J Res Med Sci. 2015 Aug;20(8):751-6. doi: 10.4103/1735-1995.168377.

4. Chen D, Chen G, Jiang W, et al. Association of the Collagen Signature in the Tumor Microenvironment With Lymph Node Metastasis in Early Gastric Cancer. JAMA Surg. 2019 Mar 1;154(3):e185249. doi: 10.1001/jamasurg.2018.5249.

5. Tekpli X, Lien T, Røssevold AH, et al. An independent poor-prognosis subtype of breast cancer defined by a distinct tumor immune microenvironment. Nat Commun. 2019 Dec 3;10(1):5499. doi: 10.1038/s41467-019-13329-5.

6. Stakheyeva M, Riabov V, Mitrofanova I, et al. Role of the Immune Component of Tumor Microenvironment in the Efficiency of Cancer Treatment: Perspectives for the Personalized Therapy. Curr Pharm Des. 2017;23(32):4807-4826. doi: 10.2174/1381612823666170714161703.

7. Yamamoto K, Makino T, Sato E, et al. Tumor-infiltrating M2 macrophage in pretreatment biopsy sample predicts response to chemotherapy and survival in esophageal cancer. Cancer Sci. 2020 Apr;111(4):1103-1112. doi: 10.1111/cas.14328. Epub 2020 Feb 18.

8. Newman AM, Liu CL, Green MR, et al. Robust enumeration of cell subsets from tissue expression profiles. Nat Methods. 2015 May;12(5):453-7. doi: 10.1038/nmeth.3337. 
9. Jiang P, Gu S, Pan D, et al. Signatures of T cell dysfunction and exclusion predict cancer immunotherapy response. Nat Med. 2018 Oct;24(10):1550-1558. doi: 10.1038/s41591-018-0136-1.

10. Eisenhauer EA, Therasse $P$, Bogaerts $J$, et al. New response evaluation criteria in solid tumours: revised RECIST guideline (version 1.1). Eur J Cancer. 2009 Jan;45(2):228-47. doi: 10.1016/j.ejca.2008.10.026.

11. Litviakov N, Tsyganov M, Larionova I, et al. Expression of M2 macrophage markers YKL-39 and CCL18 in breast cancer is associated with the effect of neoadjuvant chemotherapy. Cancer Chemother Pharmacol. 2018 Jul;82(1):99-109. doi: 10.1007/s00280-018-3594-8.

12. Yoshimura A, Naka T, Kubo M. SOCS proteins, cytokine signalling and immune regulation. Nat Rev Immunol. 2007 Jun;7(6):454-65. doi: 10.1038/nri2093.

13. Fujimoto M, Naka T. SOCS1, a Negative Regulator of Cytokine Signals and TLR Responses, in Human Liver Diseases. Gastroenterol Res Pract. 2010;2010:470468. doi: 10.1155/2010/470468.

14. Yoshimura A, Ohishi HM, Aki D, et al. Regulation of TLR signaling and inflammation by SOCS family proteins. J Leukoc Biol. 2004 Mar;75(3):422-7. doi: 10.1189/jlb.0403194. Epub 2004 Jan 14.

15. Blasius AL, Beutler B. Intracellular toll-like receptors. Immunity. 2010 Mar 26;32(3):305 - 15. doi: 10.1016/j.immuni.2010.03.012.

16. Aderem A, Ulevitch RJ. Toll-like receptors in the induction of the innate immune response. Nature. 2000 Aug 17;406(6797):782-7. doi: 10.1038/35021228.

17. Mansell A, Smith R, Doyle SL, et al. Suppressor of cytokine signaling 1 negatively regulates Toll-like receptor signaling by mediating Mal degradation. Nat Immunol. 2006 Feb;7(2):148-55. doi: 10.1038/ni1299. Epub 2006 Jan 15. PMID: 16415872.

18. Starr R, Willson TA, Viney EM, et al. A family of cytokine-inducible inhibitors of signalling. Nature. 1997 Jun 26;387(6636):917-21. doi: 10.1038/43206.

19. Nicholson SE, Hilton DJ. The SOCS proteins: a new family of negative regulators of signal transduction. J Leukoc Biol. 1998 Jun;63(6):665-8. doi: 10.1002/jlb.63.6.665.

20. Nakagawa R, Naka T, Tsutsui H, et al. SOCS-1 participates in negative regulation of LPS responses. Immunity. 2002 Nov;17(5):677-87. doi: 10.1016/s1074-7613(02)00449-1.

21. Endo TA, Masuhara $M$, Yokouchi $M$, et al. A new protein containing an SH2 domain that inhibits JAK kinases. Nature. 1997 Jun 26;387(6636):921-4. doi: 10.1038/43213.

22. Komazaki T, Nagai H, Emi M, et al. Hypermethylation-associated inactivation of the SOCS-1 gene, a JAK/STAT inhibitor, in human pancreatic cancers. Jpn J Clin Oncol. 2004 Apr;34(4):191-4. doi: 10.1093/jjco/hyh035.

23. Chen CY, Tsay $\mathrm{W}$, Tang JL, et al. SOCS1 methylation in patients with newly diagnosed acute myeloid leukemia. Genes Chromosomes Cancer. 2003 Jul;37(3):300-5. doi: 10.1002/gcc.10222.

24. Yoshikawa H, Matsubara K, Qian GS, et al. SOCS-1, a negative regulator of the JAK/STAT pathway, is silenced by methylation in human hepatocellular carcinoma and shows growth-suppression activity. Nat Genet. 2001 May;28(1):29-35. doi: 10.1038/ng0501-29. 
25. McCormick SM, Gowda N, Fang JX, et al. Suppressor of Cytokine Signaling (SOCS) 1 Regulates Interleukin-4 (IL-4)-activated Insulin Receptor Substrate (IRS)-2 Tyrosine Phosphorylation in Monocytes and Macrophages via the Proteasome. J Biol Chem. 2016 Sep 23;291(39):20574-87. doi: 10.1074/jbc.M116.746164.

26. Whyte CS, Bishop ET, Rückerl D, et al. Suppressor of cytokine signaling (SOCS) 1 is a key determinant of differential macrophage activation and function. J Leukoc Biol. 2011 Nov;90(5):845-54. doi: 10.1189/jlb.1110644. Epub 2011 May 31.

27. Liu Y, Stewart KN, Bishop E, et al. Unique expression of suppressor of cytokine signaling 3 is essential for classical macrophage activation in rodents in vitro and in vivo. J Immunol. 2008 May 1;180(9):6270-8. doi: 10.4049/jimmunol.180.9.6270.

28. Mantovani A, Sica A, Sozzani S, et al. The chemokine system in diverse forms of macrophage activation and polarization. Trends Immunol. 2004 Dec;25(12):677-86. doi: 10.1016/j.it.2004.09.015.

29. Cui X, Morales RT, Qian W, et al. Hacking macrophage-associated immunosuppression for regulating glioblastoma angiogenesis. Biomaterials. 2018 Apr;161:164-178. doi: 10.1016/j.biomaterials.2018.01.053.

30. Wen ZF, Liu H, Gao R, et al. Tumor cell-released autophagosomes (TRAPs) promote immunosuppression through induction of M2-like macrophages with increased expression of PD-L1. J Immunother Cancer. 2018 Dec 18;6(1):151. doi: 10.1186/s40425-018-0452-5.

31. Park JH, van Wyk H, McMillan DC, et al. Preoperative, biopsy-based assessment of the tumour microenvironment in patients with primary operable colorectal cancer. J Pathol Clin Res. 2020 Jan;6(1):30-39. doi: 10.1002/cjp2.143.

32. Anitei MG, Zeitoun G, Mlecnik B, et al. Prognostic and predictive values of the immunoscore in patients with rectal cancer. Clin Cancer Res. 2014 Apr 1;20(7):1891-9. doi: 10.1158/1078-0432.CCR13-2830.

\section{Figures}


MCPcounter was used to evaluate abundance of immune cells in tumor tissue of TCGA data set.

Abundance of M2 macrophage was strongly associated with DSS in TCGA ESCA data set.

SOCS1 and ORAI3 were verified in clinical specimens respectively.
279 genes were selected as potential functional genes of M2 macrophages in TCGA ESCA data set.

11 genes that had significant predictive value of nCRT response were finally selected in GES45670 dataset.

SOCS1and ORAI3 that had largest absolute regression coefficient were finally selected for clinical verification.
Assess the predictive value of Score in GES45670 dataset and GSE104958 dataset which were used for training set and validation set.
The Score for predicting response of nCRT was defined as the sum of the two genes' regression coefficients multiplying by ratios of the expression of the two genes to the abundance of M2 macrophage genes.

Figure 1

The flow chart of analysis. 

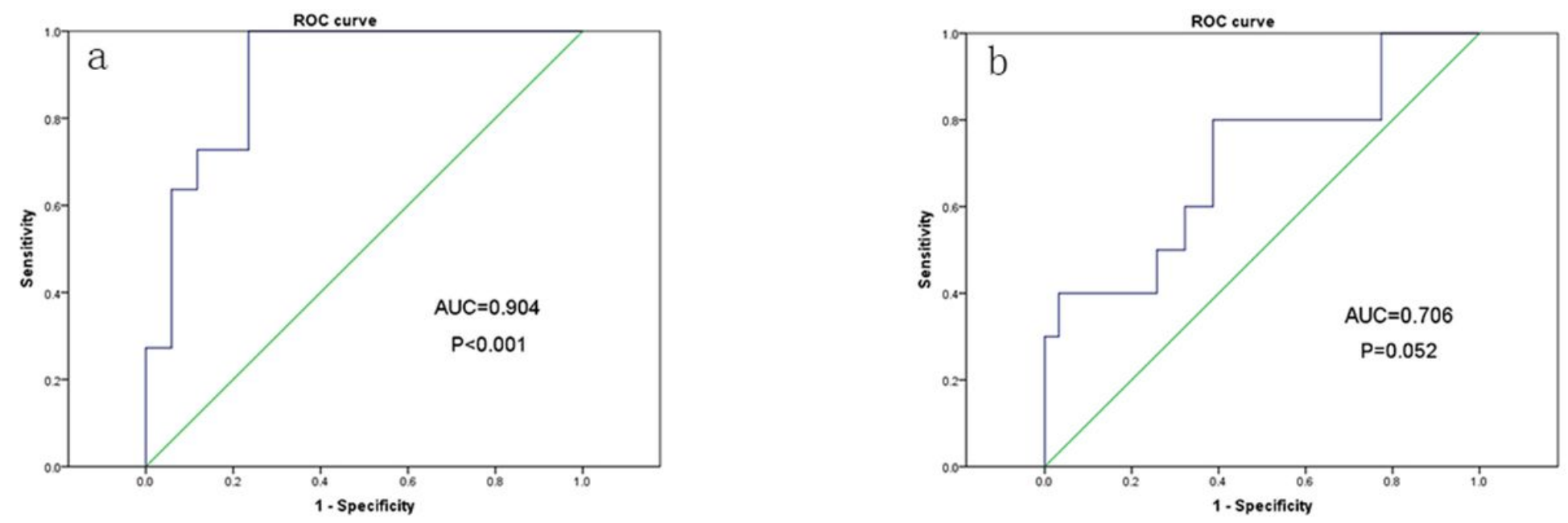

Figure 2

The predictive efficacy of score in the training set and validation set for neoadjuvant therapy. Figure $2 \mathrm{a}$ The score of ROC curve in the training set GSE45670 (AUC $=0.904,95 \% \mathrm{Cl}: 0.792-1, \mathrm{P}<0.001$ ). Figure $2 \mathrm{~b}$ The score of ROC curve in the validation set GSE104958 (AUC $=0.706,95 \% \mathrm{Cl}: 0.5033-0.9096, P=0.052$ ).
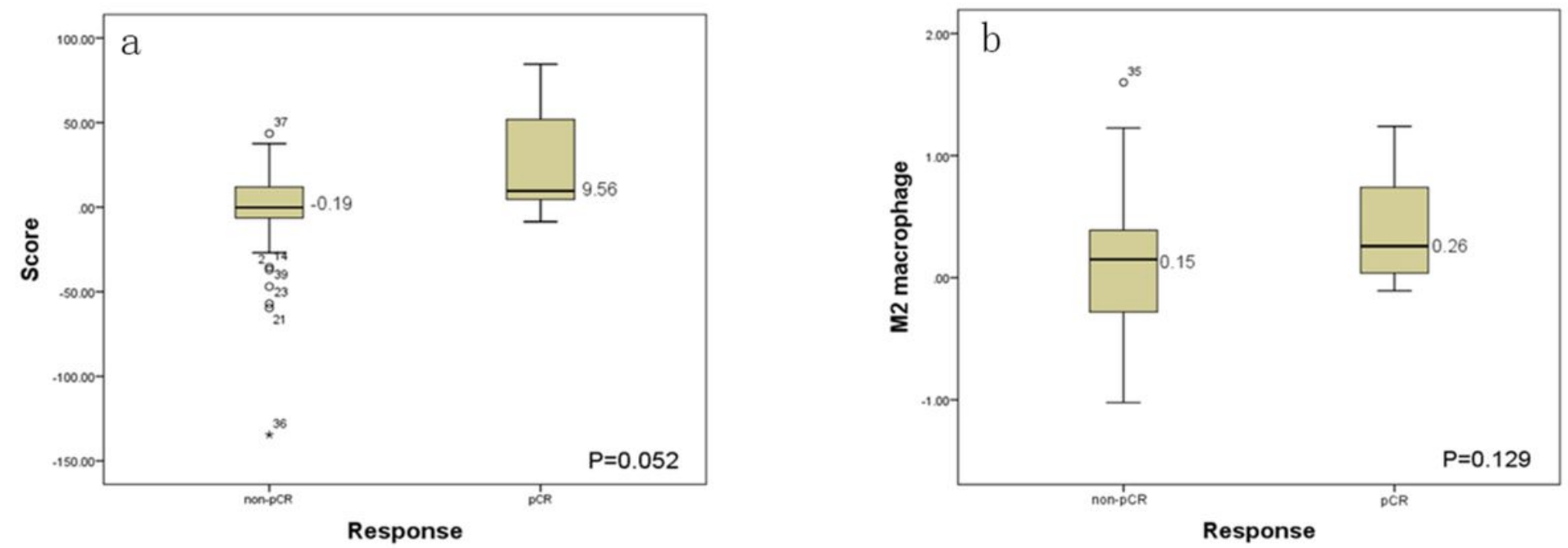

\section{Figure 3}

The correlation between score and M2 macrophage infiltration abundance in GSE104958 and neoadjuvant response. Figure $3 a$ The Score of patients with PR in remission was higher than that of patients with $P D+S D$ in remission, with the median values of -0.19 and $9.56(P=0.052)$, respectively. Figure $3 \mathrm{~b}$ There was no significant difference in $\mathrm{M} 2$ infiltration abundance between PR patients and $P D+S D$ patients, with the median value of 0.15 and $0.26(P=0.129)$, respectively. 

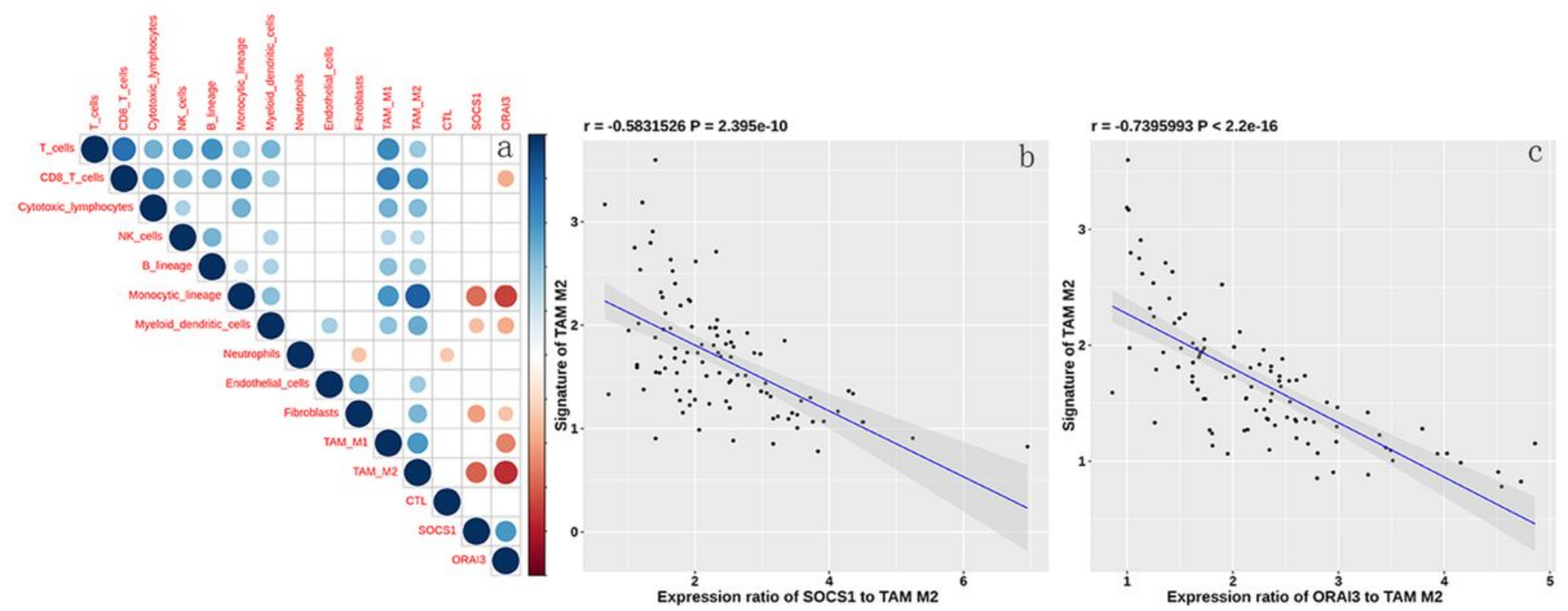

Figure 4

The correlations between the infiltration abundance of M2 macrophage and the expression of SOCS1 and the expression of ORAI3. Figure 4a The correlations between the infiltration abundance of immune cells and expression of SOCS1 and the expression of ORAI3 (red represents negative correlation and blue represents positive correlation). Figure $4 \mathrm{~b}$ The negative correlation between the expression ratio of SoCS1 to M2 macrophage, namely Score 1, and the infiltration abundance of M2 macrophage from the regression analysis scatter diagram. Figure $4 c$ The negative correlation between the expression ratio of ORAI3 to M2 macrophage, namely Score 2, and the infiltration abundance of M2 macrophage from the regression analysis scatter diagram. 

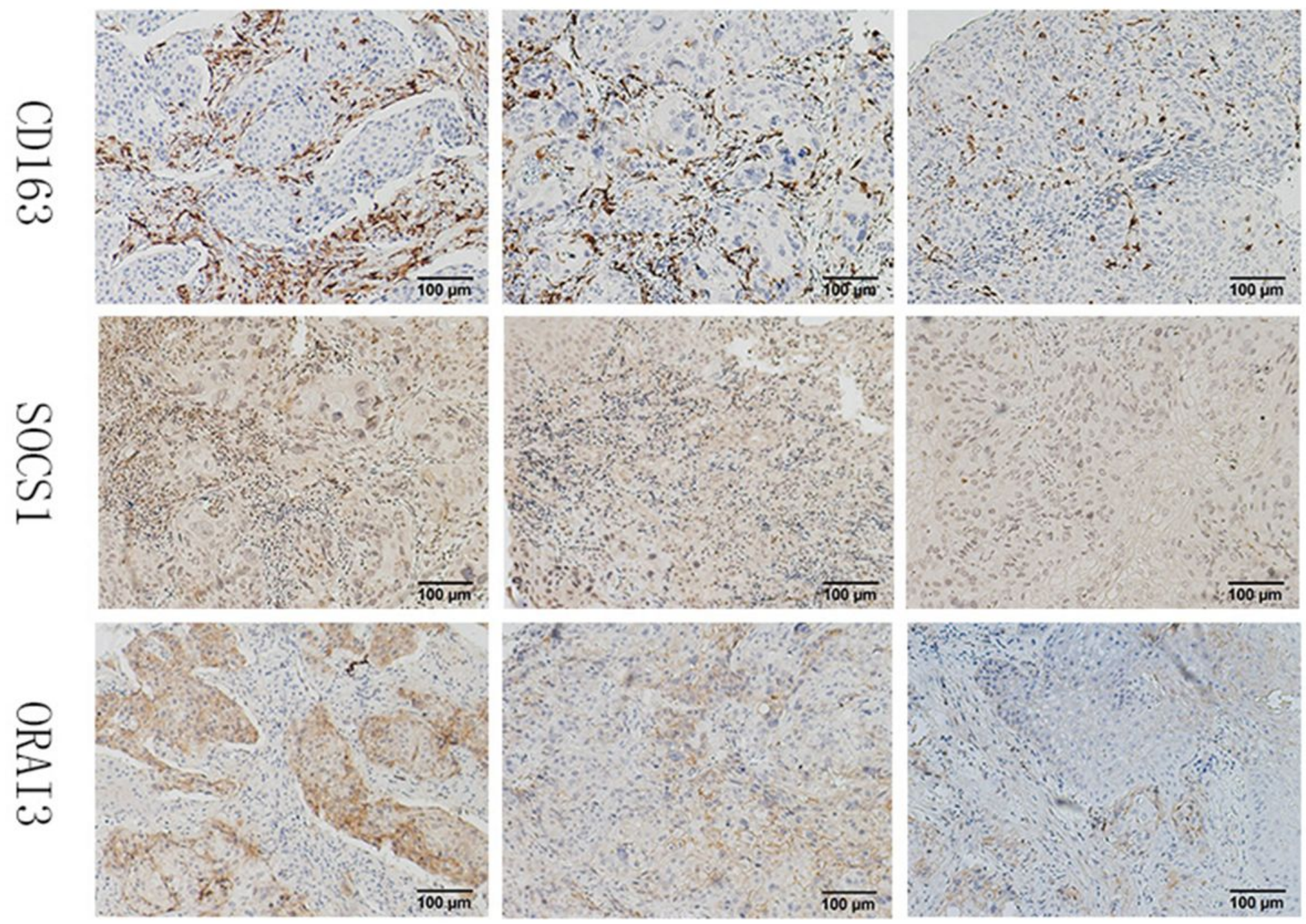

\section{Figure 5}

Example images of immunohistochemical staining. The H-scores of CD163 expression on the membrane of M2 macrophages were 270, 210 and 90 from left to right; of SOCS1 expression on nucleus and cytoplasm were 80, 30 and 10; of ORAI3 expression on the membrane were 120, 50 and 20.
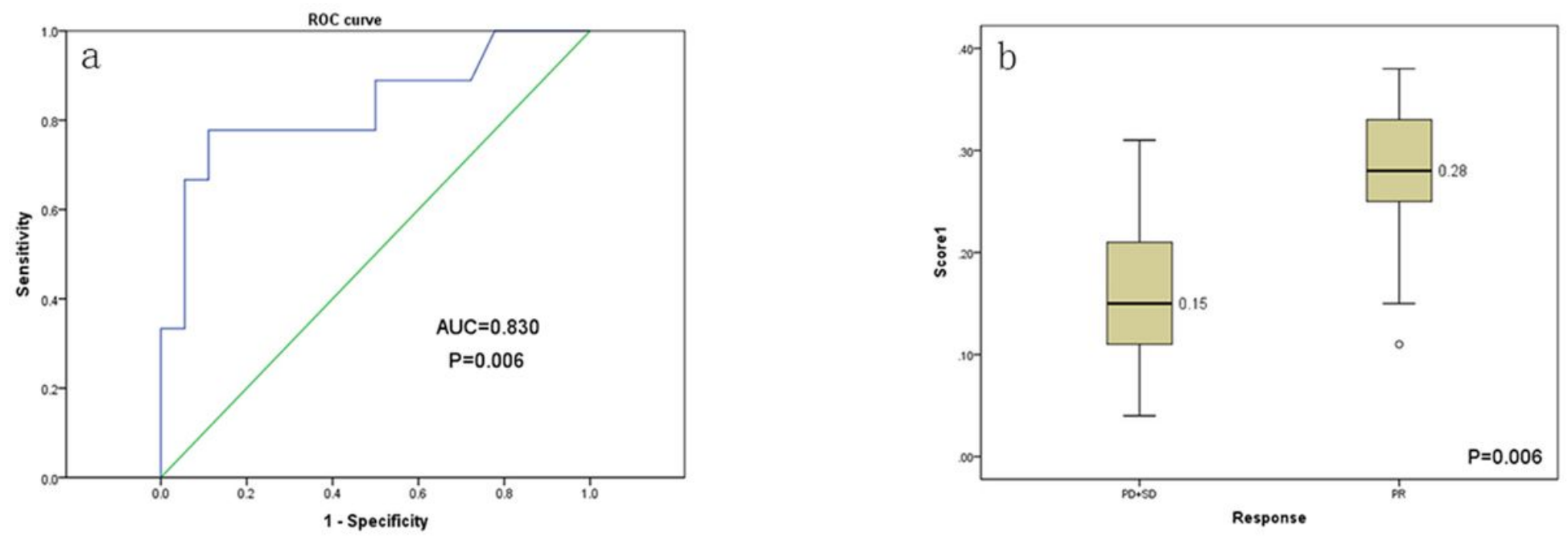
Figure 6

The predictive power of Score 1 for neoadjuvant efficacy in clinical samples. Figure 6a. The score 1 of ROC curve in predicting neoadjuvant efficacy of esophageal squamous cell carcinoma (AUC $=0.830,95 \%$ Cl: $0.647-1, P=0.006)$. Figure $6 \mathrm{~b}$ The correlation between remission and Score1. The score 1 of patients with PR was significantly higher than that of patients with PD+SD (median 0.28 and $0.15, P=0.006$ ).

\section{Supplementary Files}

This is a list of supplementary files associated with this preprint. Click to download.

- SupplementaryTable1.xlsx

- SupplementaryTable2.xlsx

- SupplementaryTable3.xlsx 